\section{Long-term Remission of Wells Syndrome With Omalizumab}

Coattrenec $\mathrm{Y}^{1}$, Ibrahim Yasmine $\mathrm{L}^{2}$, Harr $\mathrm{T}^{1}$, Spoerl D ${ }^{1 *}$, Jandus $\mathrm{P}^{1 *}$ ${ }^{I}$ Division of Immunology and Allergology, Department of Internal Medicine, University Hospital and Medical Faculty, Geneva, Switzerland

${ }^{2}$ Division of Clinical Pathology, University Hospital and Medical Faculty, Geneva, Switzerland

*DS and PJ contributed equally to this work.

J Investig Allergol Clin Immunol 2020; Vol. 30(1): 58-59 doi: 10.18176/jiaci.0436

Key words: Wells syndrome. Eosinophilic cellulitis. Omalizumab. Successful treatment.

Palabras clave: Síndrome Wells. Celulitis eosinofílica. Omalizumab. Tratamiento eficaz.

Eosinophilic cellulitis (Wells syndrome) is an uncommon recurrent inflammatory dermatosis. It is characterized by wide clinical variability comprising itching and/or burning tender erythematous lesions, sometimes with urticaria, vesicles and/or bullae, and granulomatous eosinophilic infiltrates in the dermis. The condition was first described by Wells in 1971, and, to date, fewer than 200 cases have been reported in the literature. The histological features are dependent on the stage of the disease, although dermal edema, marked eosinophilic infiltrate without signs of vasculitis, and flame figures are characteristic. Peripheral eosinophilia is frequent [1].

We report the case of a 67-year-old man with history of recurring episodes of erythema lasting 1-3 days, as well as occasional concomitant edema of the limbs, tongue, and face occurring approximately every 2 weeks for the last 30 years. No trigger factors, including drugs, could be identified. Values for complete white cell blood count (including eosinophils), tryptase, functional C1-inhibitor esterase, and C4 were within the normal range. A computed tomography scan of the thorax and abdomen was unremarkable, with no signs of malignancy or inflammatory lesions. Analysis of lymphocyte populations in peripheral blood using flow cytometry did not reveal a monoclonal lymphocytic population. Autoimmune testing for antinuclear antibodies and extractable nuclear antigens was negative. Parasitic infections were excluded by stool and serological tests. Prick tests and specific IgE for food allergy were negative, and total $\operatorname{IgE}$ was not elevated. A skin biopsy of the arm performed in 2007 revealed a dense dermal perivascular and interstitial lymphocytic and histiocytic inflammatory infiltrate associated with abundant eosinophils and flame figures compatible with Wells syndrome. Initially, the patient was treated with antihistamines at up to 4-fold higher than the licensed dose, then with topical and systemic corticosteroids, followed by azathioprine up to $150 \mathrm{mg}$ daily, tranexamic acid up to $1000 \mathrm{mg} 3$ times daily, and, finally, a gluten-free diet. None of these approaches was successful. Despite eradication of Helicobacter pylori, the recurrent skin lesions and edema persisted. The patient was lost to follow-up and treated by various doctors. Detailed data are not available. He eventually came to our clinic 10 years later in 2017 because of exacerbation of skin symptoms while receiving antihistamines and topical corticosteroids.

A new skin biopsy (Figure) revealed a dermal infiltrate of perivascular and interstitial lymphocytes and histiocytes with

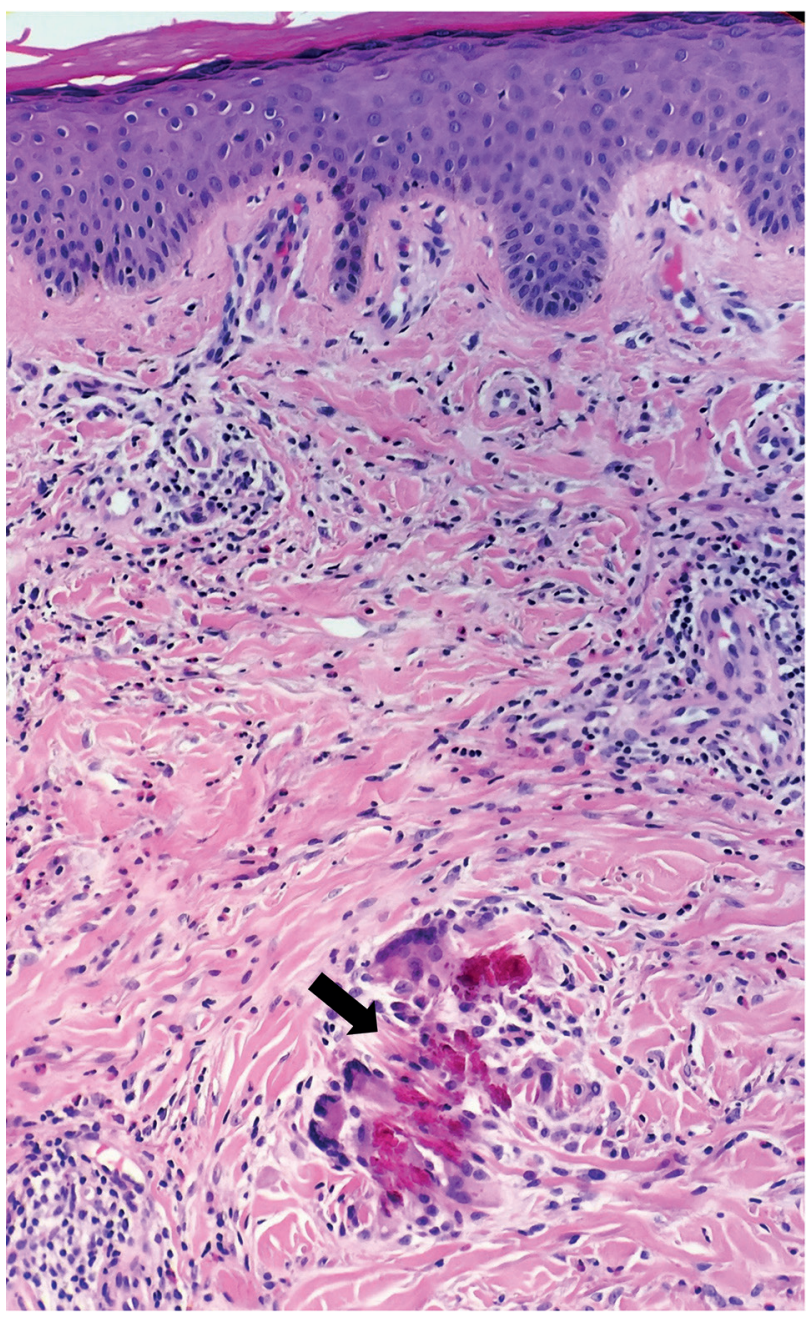

Figure. Skin biopsy with marked dermal eosinophilic infiltrate, some multinucleate giant cells, and typical flame figures (arrow) (hematoxylineosin, original magnification $\times 200$ ) 
numerous eosinophils, some multinucleate giant cells, and typical flame figures. At this time, recurrent edema and strong pruritus led us to suspect concomitant mast cell activation. Therapy was started with subcutaneous omalizumab $300 \mathrm{mg}$ per month. After a few days, the skin manifestations improved dramatically, and the patient remained asymptomatic under omalizumab during a follow-up period of 24 months. We are currently trying to discontinue omalizumab by prolonging the interval between treatments. The patient is now treated every 8 weeks, with no signs of recurrence. Complete discontinuation will be attempted in the next 6 months.

The pathogenesis of Wells syndrome remains unknown. One hypothesis is that it is caused by a nonspecific hypersensitivity reaction to endogenous or exogenous stimuli such as infection, vaccination, medication, insect bites, myeloproliferative disorders, or malignant disorders. However, despite extensive investigations, it is not often possible to identify a trigger factor. Dysregulation of tissue eosinophilia with high IL-5 production may also play a role in pathogenesis $[2,3]$.

Multiple treatment regimens have been used, with variable success rates. Given the rarity of the disease, no randomized placebo-controlled clinical trials have been conducted to date. In general, if a trigger factor cannot be detected or excluded, the first-line treatments are topical or systemic corticosteroids and antihistamines. If the disease recurs, immunomodulatory or immunosuppressive therapies such as dapsone and cyclosporine can be used. Other alternative treatments include azathioprine, antimalarial drugs, tacrolimus, interferon $\alpha$ and $\gamma$, anti-TNF $\alpha$, and colchicine [4].

In 2018, Egeland et al [5] published the first case of a patient successfully treated with omalizumab. This drug is well established as second-line treatment of chronic spontaneous urticaria. Omalizumab binds free IgE, resulting in downregulation of expression of the high affinity $\operatorname{IgE}$ receptor (FceRI) on mast cells and basophils, thus preventing their activation and generating a beneficial effect on the development of urticaria [6]. Other potential mechanisms of action of omalizumab have been reported. Eosinophils have been shown to play a role in a multitude of inflammatory and autoimmune diseases. Upregulation of FceRI on skin-infiltrating eosinophils has been reported in atopic dermatitis [7] and in bullous pemphigoid [8]. A small number of patients with these skin diseases have been successfully treated with omalizumab $[9,10]$. Therefore, we assume that the skin eosinophils in Wells syndrome upregulate FceRI, which is subsequently downregulated with omalizumab. This would explain the beneficial effect, as previously described in atopic dermatitis, bullous pemphigoid, and, in particular, chronic spontaneous urticaria. However, our hypothesis is speculative and needs to be proven in studies that characterize FceRI on the eosinophils of patients with Wells syndrome receiving treatment.

According to the literature, the case we report is the second to date of Wells syndrome that responded successfully to omalizumab. Follow-up in the present case was longer than in the previous reported case. Although the mechanism of action of omalizumab is not understood in Wells syndrome, this case demonstrates that omalizumab might be an effective alternative in refractory disease.

\section{Funding}

The authors declare that no funding was received for the present study.

\section{Conflicts of Interest}

The authors declare that they have no conflicts of interest.

\section{References}

1. Long $H$, Zhang $G$, Wang L, Lu Q. Eosinophilic Skin Diseases: $A$ Comprehensive Review. Clin Rev Allergy Immunol. 2016;50(2): 189-213.

2. Yagi H, Tokura Y, Matsushita K, Hanaoka K, Furukawa F, Takigawa M. Wells' syndrome: a pathogenic role for circulating CD4+CD7- T cells expressing interleukin-5 mRNA. Br J Dermatol. 1997;136(6):918-23.

3. Mashima E, Sawada Y, Yamaguchi T, Ohmori S, Haruyama S, Yoshioka M, et al. Eosinophilic Cellulitis Possibly Due to Mosquito Bite With High IL-5 Production. J Investig Allergol Clin Immunol. 2017;27(2):149-50.

4. Rassler F, Lukacs J, Elsner P. Treatment of eosinophilic cellulitis (Wells syndrome) - a systematic review. J Eur Acad Dermatol Venereol. 2016;30(9):1465-79.

5. Egeland O, Balieva F, Undersrud E. Wells syndrome: a case of successful treatment with omalizumab. Int J Dermatol. 2018;57(8):994-5.

6. Kaplan AP, Gimenez-Arnau AM, Saini SS. Mechanisms of action that contribute to efficacy of omalizumab in chronic spontaneous urticaria. Allergy. 2017;72(4):519-33.

7. Tanaka Y, Takenaka M, Matsunaga Y, Okada S, Anan S, Yoshida $\mathrm{H}$, et al. High affinity IgE receptor (Fc epsilon RI) expression on eosinophils infiltrating the lesions and mite patch tested sites in atopic dermatitis. Arch Dermatol Res. 1995;287(8):712-7.

8. Messingham KN, Holahan HM, Frydman AS, Fullenkamp C, Srikantha R, Fairley JA. Human eosinophils express the high affinity IgE receptor, FcepsilonRI, in bullous pemphigoid. PLOS One. 2014;9(9):e107725.

9. Balakirski G, Alkhateeb A, Merk HF, Leverkus M, Megahed M. Successful treatment of bullous pemphigoid with omalizumab as corticosteroid-sparing agent: report of two cases and review of literature. J Eur Acad Dermatol Venereol. 2016;30(10):1778-82.

10. Holm JG, Agner T, Sand C, Thomsen SF. Omalizumab for atopic dermatitis: case series and a systematic review of the literature. Int J Dermatol. 2017;56(1):18-26.

I Manuscript received March 18, 2019; accepted for publication July 23, 2019.

Peter Jandus

Division of Immunology and Allergology Department of Internal Medicine University Hospital and Medical Faculty Rue Gabrielle Perret-Gentil 4 1211 Geneva 14, Switzerland E-mail: peter.jandus@hcuge.ch 